\title{
General Psychiatry Concomitant atopic dermatitis and narcolepsy type 1: psychiatric implications and challenges in management
}

\author{
Justin Chin (D) , ${ }^{1}$ Craig Bearison, ${ }^{2}$ Nanette Silverberg, ${ }^{3}$ Mary Lee Wong ${ }^{4,5}$
}

To cite: Chin J, Bearison C, Silverberg N, et al. Concomitant atopic dermatitis and narcolepsy type 1: psychiatric implications and challenges in management. General Psychiatry 2019;32:e100094. doi:10.1136/ gpsych-2019-100094

Received 25 May 2019 Revised 12 August 2019 Accepted 06 September 2019

Check for updates

(c) Author(s) (or their employer(s)) 2019. Re-use permitted under CC BY-NC. No commercial re-use. See rights and permissions. Published by BMJ.

${ }^{1}$ Department of Primary Care, Touro College of Osteopathic Medicine, New York City, New York, USA

${ }^{2}$ Department of Internal Medicine, Beth Israel Medical Center, New York City, New York, USA

${ }^{3}$ Department of Pediatric Dermatology, Mount Sinai Health System, New York City, New York, USA

${ }^{4}$ Department of Allergy and Immunology, Beth Israel Medical Center, New York City, New York, USA

${ }^{5}$ Department of Medicine, Icahn School of Medicine at Mount Sinai, New York City, New York, USA

Correspondence to

Mr Justin Chin;

jchin2@student.touro.edu

\section{ABSTRACT}

Atopic dermatitis (AD) and narcolepsy type 1 (NT1) are two distinct diseases that have not been classically shown to be related. The potential connection between the known immunological aetiology of $\mathrm{AD}$ and the proposed autoimmune pathophysiology of dysregulation in NT1; however, is the subject of ongoing speculation and debate with advances in gene sequencing and technology. Here, we present a case of a patient with concomitant refractory $A D$ and NT1 and review the current research on their immunological relationship and the challenges in management relative to disease burden and psychiatric comorbidities.

\section{INTRODUCTION}

Narcolepsy is traditionally classified into two types, fittingly named narcolepsy type 1 (NT1) and NT2. The pathophysiology of both types has been shown to involve the loss of orexin-producing neurons in the lateral hypothalamus, but differ in the physical manifestation as NT1 is characterised by cataplexy while NT2 is not. Cataplexy is the sudden and transient loss of consciousness, typically triggered by a strong emotion and without a subsequent postictal period. The mechanism for orexin-producing neurons loss is not well understood, with autoimmune and neurological dysfunctions among the most widely researched aetiologies for NT1. ${ }^{1}$

In recent gene studies, a strong association has been found between NT1 and the human leucocyte antigen (HLA) haplotype DQ beta-1 (DQB1)*06:02, with over $90 \%$ of patients having this haplotype. This polymorphism occurs in the T-cell receptor alpha locus, which affects VJ2 recombination and creating a T-cell haplotype complex that triggers autoimmunity against orexin-producing neurons. ${ }^{2}$ Higher levels of antibodies against Trib2 (expressed on orexin-secreting neurons among other neuron types) have been found in patients with NT1, which in turn have been positively correlated to increasing cataplexy severity. ${ }^{13}$
Increased tumour necrosis factor (TNF) and interleukin-6 serum levels have been found in patients with narcolepsis compared with that in age-matched and gender-matched controls, which suggests that cytokine levels themselves may have a role in the regulation of sleep, as stimulatory drugs were associated with lower TNF levels. ${ }^{14}$ Despite the increasing body of evidence for an autoimmune connection, there are no narcolepsy-specific antibodies, known associations between NT1 with nuclear protein autoantibodies (such as antinuclear antibodies (ANA), anti-double stranded deoxyribonucleic acid (anti-dsDNA), etc), or increase in immunoglobulin $\mathrm{G}$ index or oligoclonal bands in the cerebral spinal fluid of NT1 patients. ${ }^{1-3}$

Along similar autoimmune pathways to NT1, defects in the innate and adaptive immune response in conjunction with epidermal barrier dysfunction are implicated in the pathogenesis of atopic dermatitis (AD). Reduced toll-like receptor-2 (TLR-2) and TLR-9 function observed in some patients with $\mathrm{AD}$ is thought to lead to epidermal hyperplasia and impairment in the skin's barrier function via a decreased release of various inflammatory mediators. ${ }^{5}$

$\mathrm{AD}$ is associated with sensitisation to aeroallergen or food allergens, with most evidence indicating a role for immunoglobulin $\mathrm{E}$ (IgE)-mediated allergies in modulating the severity of $\mathrm{AD} .{ }^{5}{ }^{6}$ Given the suspicion for immune dysregulation in the aetiology and/or physiology of narcolepsy, there has also been interest in elucidating a potential connection or similarity between narcolepsy and atopic or autoimmune disease pathology. ${ }^{5-7}$

We present a patient with a history of NT1 and $\mathrm{AD}$ who presented to an allergy clinic for management of food and environmental allergies, while also reviewing the psychiatric sequelae that may be associated with poor disease management. 

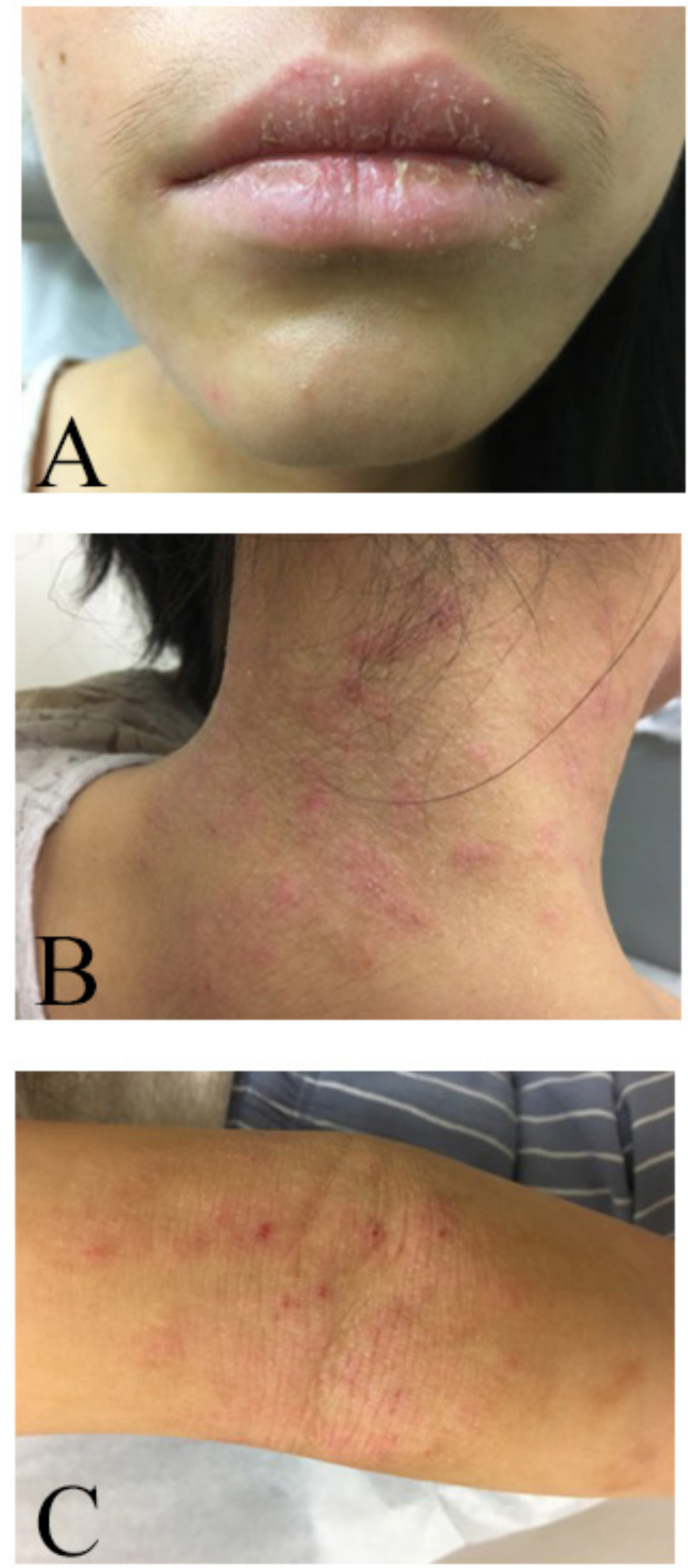

Figure 1 (A-C) Photos of patient's lips (A), nape (B) and right antecubital fossa $(C)$. Photos taken during patient's initial presentation to the allergy clinic.

\section{CASE HISTORY}

A 19-year-old Asian American female with NT1 and AD presented to the allergy clinic for evaluation of food and environmental allergies. Her medical history was significant for diagnosis of $\mathrm{AD}$ at age 9 , in which shortly after, she began having symptoms suggestive of narcolepsy, with excessive daytime fatigue, occasional hypnogogic hallucinations and sleep paralysis. She did not mention her sleep symptoms until the age of 16 and was formally diagnosed with NT1 when she developed increasingly excessive daytime sleepiness followed by cataplexy several months later. Coupled with her clinical presentation, recent polysomnogram and multiple sleep latency tests further supported the diagnosis of NT1 with short rapid eye movement latency and sleep-onset rapid eye movement periods during all nap intervals, respectively. Since her NT1 diagnosis, the patient has reported sleeping an average of 6 hours each night with two daytime naps. She has previously been prescribed stimulants such as modafinil, methylphenidate and amphetamine salts, but is following with her primary care on creating a sustainable management regimen.

Her AD has been conservatively managed for several years with minimal improvement on dietary control, topical corticosteroids, tacrolimus and over the counter emollients. The pruritus associated with her eczematous lesions, particularly on her antecubital regions, have frequently disrupted her sleep, with intense flares-up occurring every 3-4 months (figure 1 ). She had attributed these flare-ups with environmental allergies and food sensitivities but has never been formally identified. The patient denies any history of asthma or allergic rhinitis, with crisaborole as the sole pharmacological treatment of her AD. For management of sleep disruption secondary to pruritus, the patient has trialled several first-generation and second-generation antihistamines but all were discontinued secondary to increased daytime somnolence and worsening of her narcolepsy symptoms. She endorsed frequent feelings of depression and anxiety secondary to poor management of her medical illnesses but denies any thoughts of suicidality or homicidality. Patient Health Questionaire-2 was negative and mild anxiety was noted on the Hamilton Anxiety Scale (score of 14).

Laboratory values were significant for serum IgE of 982 and radioallergosorbent test positive for dust mites (including Dermatophagoides pteronyssinus and D. farinae), common birch, hazelnut, cat dander and several other antigens. Of note, the patient has tested positive for HLA DQB1*06:02. She has no family history of autoimmune diseases or atopy. She tested negative for ANA, anti-dsDNA, rheumatoid factor, anti-Smith antibody, anti-Ro, anti-La and anticyclic citrullinated peptide and her C-reactive protein, erythrocyte sedmintation rate, thyroid stimulating hormone and haemoglobin A1c were all within normal limits. Physical examination revealed thick, erythematous oozing plaques of the antecubital regions and the neck. On assessment, she had an Eczema Area and Severity Index score of 3.45 and Scoring Atopic Dermatitis score of 37 .

\section{DISCUSSION}

The comorbidity of eczema and narcolepsy creates a positive feedback loop that has exacerbated each of 
these conditions in our patient. Practising good sleep hygiene and maintaining a normal sleep-wake cycle are important tools in the management of narcolepsy. Our patient reported frequent nighttime sleep disruptions secondary to eczema-induced pruritus. Antihistamines, such as diphenhydramine, are one of the first-line treatments for pruritus and sleep disruption in patients with eczema. Medications with sedative properties; however, are discouraged in patients with narcolepsy, as they can have deleterious effects on sleep hygiene. Our patient endorsed this phenomenon, as she could not tolerate antihistaminic therapy. Through greater permeability of the blood-brain barrier, patients with $\mathrm{AD}$ can be more susceptible to the side effect profile of antihistamines, especially when combined with other comorbid conditions such as NT1. Without adequate management of pruritus, this patient experienced perpetuation of the itch-scratch cycle, worsening both her eczema and narcoleptic symptoms as she noticed increased itching and areas of $\mathrm{AD}$ with greater daytime fatigue. She developed and self-reported severe anxiety secondary to her poor symptomatic control, which further exacerbated this positive feedback loop as anxiety can be an additional trigger for $\mathrm{AD}$ flares. ${ }^{8}$ She was constantly concerned with when her next flare-up may be and how it would affect her daily life.

The patient tested positive for several environmental and food allergies, most notably dust mites. Dust mites are the most common allergen known to aggravate $\mathrm{AD}$, and avoidance can greatly reduce exacerbations and long-term sequelae. ${ }^{9}$ With the patient's long history of $\mathrm{AD}$ and its refractory nature to standard interventions, reduction of allergen exposure had only marginally improved her symptoms. Options such as allergen-specific immunotherapy (SIT) against dust mite species were discussed; however, immunotherapy has been cautioned in patients with autoimmune conditions, as no studies to date have evaluated the safety of allergen-specific immunotherapy in patients with comorbid autoimmune conditions. ${ }^{6} 8$ More research is needed to evaluate an immunological mechanism of NT1 and any potential overlap with atopic or autoimmune disease. Efforts to elucidate the immunopathogenesis of disease should be done in conjunction with trials for development of new immunotherapeutic interventions. Thus far, case series and longitudinal follow-ups of patients receiving immune-targeted treatments such as intravenous immunoglobulins, plasmapheresis or alemtuzumab have been conflicting or inconclusive. $^{679}$

Anxiety is a common comorbidity for both of these conditions, as data from large surveys and research databases has shown significantly higher rates of anxiety and depression in patients with each disorder independently. ${ }^{910}$ The burden can even take a toll on the medical system itself, with increased resource usage and costs associated with healthcare utilisation in dealing with psychiatric sequelae to $\mathrm{AD}$ and/or NT1. With anxiety serving as a trigger for $\mathrm{AD}$ and interfering with sleep for AT1, adjuvant psychosocial support and/or psychotropic medications are sometimes required for further management of both conditions. A multimodal, targeted approach to treatment is essential due to the potential burden associated with how these diseases can impact each other as well as the contraindications each condition presents in the treatment options for the other. Dupilumab is an interleukin-4 receptor alpha antagonist that is the only FDA-approved monoclonal antibody for the treatment of $\mathrm{AD}$ not adequately controlled with topical prescription therapies. ${ }^{8}$ Presently no data is available regarding the safety of dupilumab therapy in patients with concomitant NT1. On consultation at the clinic, the patient decided to manage her $\mathrm{AD}$ with dupilumab before revisiting her NT1 treatments. Extensive counselling was done to encourage continued follow-up with her primary care physician and sleep pathologist for management of her NT1.

\section{CONCLUSION}

$\mathrm{AD}$ and NT1 are two unique disease processes that affect different body systems. Both may share an immunological connection and various comorbidities, which can complicate treatment and management options as physicians must take into account what the standard pharmacological intervention is and how it can impact other disease processes. Future research is needed to better understand treatment options for patients with refractory eczema and comorbid autoimmune disease. In this case, and for similar cases involving multiple specialists, a multimodal, physician-patient team approach is needed to ensure optimal success. As advances in genetics and gene associations occur, this further highlights the need to reassess diseases that previously were not deemed as related, thus providing future avenues for precision medicine and patient care.

Contributors JC contributed to the literature review. CB and MLW contributed to the data collection. JC, CB and NS developed the manuscript. All authors contributed to the concept development and edited the manuscript.

Funding The authors have not declared a specific grant for this research from any funding agency in the public, commercial or not-for-profit sectors.

Competing interests None declared.

Patient consent for publication Obtained.

Provenance and peer review Not commissioned; externally peer reviewed.

Open access This is an open access article distributed in accordance with the Creative Commons Attribution Non Commercial (CC BY-NC 4.0) license, which permits others to distribute, remix, adapt, build upon this work non-commercially, and license their derivative works on different terms, provided the original work is properly cited, appropriate credit is given, any changes made indicated, and the use is non-commercial. See: http://creativecommons.org/licenses/by-nc/4.0/.

ORCID iD

Justin Chin http://orcid.org/0000-0002-3540-4596

\section{REFERENCES}

1 Mahlios J, De la Herrán-Arita AK, Mignot E. The autoimmune basis of narcolepsy. Curr Opin Neurobiol 2013;23:767-73. 
2 Fontana A, Gast H, Reith W, et al. Narcolepsy: autoimmunity, effector $T$ cell activation due to infection, or T cell independent, major histocompatibility complex class II induced neuronal loss? Brain 2010;133:1300-11.

3 Cvetkovic-Lopes V, Bayer L, Dorsaz S, et al. Elevated tribbles homolog 2-specific antibody levels in narcolepsy patients. J Clin Invest 2010;120:713-9.

4 Okun ML, Giese S, Lin L, et al. Exploring the cytokine and endocrine involvement in narcolepsy. Brain Behav Immun 2004;18:326-32.

5 Kuo I-H, Yoshida T, De Benedetto A, et al. The cutaneous innate immune response in patients with atopic dermatitis. J Allergy Clin Immunol 2013;131:266-78.
6 Aydinoz S, Huang Y-S, Gozal D, et al. Allergies and disease severity in childhood narcolepsy: preliminary findings. Sleep 2015;38:1981-4.

7 Caubet J-C, Eigenmann PA. Allergic triggers in atopic dermatitis. Immunol Allergy Clin North Am 2010;30:289-307.

8 Ellis C, Luger T, Abeck D, et al. International consensus conference on atopic dermatitis II (ICCAD II): clinical update and current treatment strategies. Br J Dermatol 2003;148(Suppl 63):3-10.

9 Forbes LR, Saltzman RW, Spergel JM. Food allergies and atopic dermatitis: differentiating myth from reality. Pediatr Ann 2009;38:84-90.

10 Ruoff CM, Reaven NL, Funk SE, et al. High rates of psychiatric comorbidity in narcolepsy: findings from the burden of narcolepsy disease (bond) study of 9,312 patients in the United States. J Clin Psychiatry 2017;78:171-6.

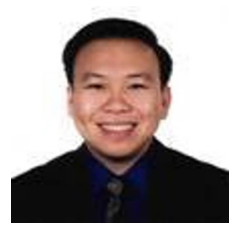

Justin Chin is a fourth year medical student at Touro College of Osteopathic Medicine in New York, USA. He completed his undergraduate degree in Integrative Biology at the University of California, Berkeley. He is the External Vice President and Chief Financial Officer for the Asian Pacific American Medical Student Association. He is also a member of the Sigma Sigma Phi and Gold Humanism Honor Society. His research interests include primarily on Asian and Pacific Islander American health issues, cultural competency, osteopathic medicine, and medical education. 